\section{Da eletrococleografia transtimpânica em pacientes com e sem hydrops endolinfático e limiares auditivos iguais ou maiores que 50 decibéis}

Letícia C. A. Soares ${ }^{1}$, Lara Silvia O. Conegundes ${ }^{1}$, Cláudia Fukuda ${ }^{2}$, Mário Sérgio L. Munhoz ${ }^{3}$

Resumo / Summary bjetivos: Por meio da análise de eletrococleografias transtimpânicas realizadas em indivíduos controles, em pacientes com diagnóstico clínico definido de bydrops endolinfático e limiares auditivos iguais ou maiores que 50 decibéis e em pacientes com labirintopatias sem evidência clínica de bydrops endolinfático e limiares auditivos iguais ou maiores que 50 decibéis, este estudo tem como objetivos determinar diferenças estatísticas entre os três grupos em relação aos parâmetros estudados; avaliar qual o parâmetro mais sensível na identificação do grupo com bydrops endolinfático e analisar se a associação de parâmetros é útil para a caracterização laboratorial do grupo com bydrops endolinfático. Forma de estudo: Clínico retrospectivo. Método: Estudo retrospectivo de 60 eletrococleografias. Foram analisadas a relação percentual entre o potencial de somação e o potencial de ação (SP/AP), a amplitude entre o primeiro pico positivo do segundo componente do potencial de ação e a linha de base (I/BSL) e a amplitude entre o segundo pico positivo do segundo componente do potencial de ação e a linha de base (II/BSL). Resultados: A análise estatística revelou comportamento diferente entre os três grupos quanto à relação SP/AP, mas não quanto aos outros dois parâmetros. $\mathrm{O}$ valor da relação SP/AP equivalente a 46,0\% apresentou índices de 75,0\% de sensibilidade e $60,0 \%$ de especificidade para a identificação do grupo com bydrops endolinfático. Conclusões: Os três grupos apresentaram comportamentos estatísticos diferentes entre si; a relação SP/AP foi o parâmetro mais sensível e específico para identificação dos pacientes com diagnóstico de bydrops endolinfático e a análise da associação dos parâmetros não foi possível.

\section{Transtympanic electrocochleography in patients with and without endolymphatic hydrops and hearing thresholds equal or major than $\mathbf{5 0}$ decibels}

Palavras-chave: eletrococleografia, perda auditiva, doença de Menière.

Key words: electrocochleography, hearing loss, Menière's disease.

\begin{abstract}
A
im: By means of electrocochleography exams performed in control subjects, in patients with endolymphatic hydrops with hearing thresholds equal or greater than 50 decibels and in patients with labyrinth disorders without clinical signs of endolymphatic hydrops with hearing thresholds equal or greater than 50 decibels, this study aimed at determining statistical differences among the three groups concerning the studied parameters, to assess what is the most sensitive parameter in the identification of the group with endolymphatic hydrops, and to analyze if the association of parameters is useful to the laboratory characterization of the group with endolymphatic hydrops. Stud design: Clinical retrospective. Method: Retrospective study of 60 electrocochleography exams. The relation of the percentage between the summating potential and the action potential (SP/AP), the amplitude between the first positive peak of the second component of the action potential and the baseline (I/BSL), and the amplitude between the second positive peak of the second component of the action potential and the baseline (II/BSL) were analyzed. Results: The analysis revealed different statistical behavior between the groups concerning the SP/AP ratio. The value of the SP/AP ratio equivalent to $46.0 \%$ presented $75.0 \%$ of sensitivity and $60.0 \%$ of specificity to the identification of the group with endolymphatic hydrops. Conclusions: The groups presented different statistical behavior among themselves; the SP/AP ratio was the most sensitive and specific parameter for the identification of the patients with endolymphatic hydrops; the analysis of the association of the parameters was not possible.
\end{abstract}

${ }^{1}$ Médica Pós-Graduanda (Mestrado) da Disciplina de Otoneurologia da Universidade Federal de São Paulo-Escola Paulista de Medicina. ${ }_{2}^{2}$ Médica Pós-Graduanda (Doutorado) da Disciplina de Otoneurologia da Universidade Federal de São Paulo-Escola Paulista de Medicina. ${ }^{3}$ Livre-docente da Disciplina de Otoneurologia da Universidade Federal de São Paulo - Escola Paulista de Medicina.

Endereço para correspondência: Letícia Clemente A. Soares - Av. 11 de junho, 1006/63 Vila Clementino 04041-003 São Paulo SP E-mail: leticiaclemente@ig.com.br

Artigo recebido em 23 de outubro de 2002. Artigo aceito em 07 de fevereiro de 2003 


\section{INTRODUÇÃO}

O bydrops endolinfático é o estado fisiopatológico no qual ocorre distensão do compartimento endolinfático. O quadro clínico da doença compreende episódios espontâneos de vertigens recorrentes, perda auditiva e zumbidos, com ou sem plenitude aural. O termo doença de Menière é aplicado aos casos de bydrops endolinfático idiopático ${ }^{1,2}$.

A eletrococleografia consiste no registro dos potenciais endococleares gerados no momento da transdução do estímulo sonoro em respostas bioelétricas e é o teste eletrofisiológico mais empregado para o diagnóstico do bydrops endolinfático. Os potenciais mais utilizados para esta finalidade são o potencial de somação (SP) que é registrado como uma pequena deflexão negativa que antecede o potencial do nervo acústico e originado dos movimentos das células ciliadas e das assimetrias vibratórias da membrana basilar e o potencial de ação (AP) que é a soma algébrica de vários potenciais de ação individuais, sendo registrado sob a forma de dois picos negativos: o primeiro denominado $\mathrm{N}_{1}$ e o segundo, de menor amplitude, denominado $\mathrm{N}_{2}$. $\mathrm{O} \mathrm{N}_{2}$ é delimitado por duas deflexões positivas (I e II) ${ }^{3}$.

A relação $\mathrm{SP} / \mathrm{AP}$ pode se alterar por aumento da amplitude do SP ou por diminuição da amplitude do AP. No bydrops endolinfático, onde a escala média encontra-se dilatada, o registro do SP é maior que o esperado. O AP é afetado pela destruição de células ciliadas e pode sofrer adaptação em doenças que envolvam o nervo auditivo e/ ou as vias auditivas do tronco encefálico, alterando também a relação $\mathrm{SP} / \mathrm{AP}^{4,5}$. Para atenuar a grande variabilidade interindividual de respostas, foi introduzida uma relação percentual entre a amplitude do SP e a amplitude do AP, tornando mais segura a avaliação da eletrococleografia ${ }^{6}$.

Nas fases avançadas do bydrops endolinfático é comum encontrarmos pacientes com limiares auditivos maiores que 50 decibéis. Estes limiares levam a uma diminuição do AP por destruição das células ciliadas e alteram a relação $\mathrm{SP} / \mathrm{AP}$, similarmente ao que ocorre nos tumores no nervo vestibular e nas doenças degenerativas, quando há diminuição do AP por adaptação ${ }^{7,8}$.

A sensibilidade e a especificidade da relação SP/AP e o incremento destes índices por meio da associação com outros parâmetros são controversos.

A análise das deflexões I e II complementaria a avaliação da eletrococleografia. Os critérios descritos para avaliação das deflexões I e II são subjetivos e pouco estudados?

Analisando a relação percentual SP/AP, a amplitude em microvolts entre o primeiro pico positivo do segundo componente do potencial de ação e a linha de base (I/BSL) e a amplitude em microvolts entre o segundo pico positivo do segundo componente do potencial de ação e a linha de base (II/BSL) por meio de ECochG TT em pacientes com diagnóstico clínico definido de bydrops endolinfático $(\mathrm{CH})$ e limiares auditivos subjetivos iguais ou maiores que 50 decibéis, em pacientes com labirintopatias sem evidência clínica de bydrops endolinfático ( $\mathrm{SH}$ ) e limiares auditivos subjetivos iguais ou maiores que 50 decibéis e em um grupo controle (CT), este estudo tem como objetivos:

1. Determinar diferenças estatísticas entre os três grupos em relação aos parâmetros estudados;

2. Avaliar qual o parâmetro mais sensível e específico na identificação do grupo com diagnóstico clínico definido de bydrops endolinfático;

3. Analisar se a associação de parâmetros é possível e útil para a caracterização laboratorial do grupo com diagnóstico clínico definido de bydrops endolinfático.

\section{MÉTODO}

O estudo foi retrospectivo, desenvolvido a partir da análise de eletrococleografias realizadas na disciplina de Otoneurologia da UNIFESP-EPM, após a aprovação pelo Comitê de Ética Médica dessa instituição. Foram incluídos no estudo 60 orelhas de 42 pacientes, divididos em três grupos. Doenças retrococleares foram afastadas em todos os pacientes. Durante a investigação, um indivíduo foi excluído por apresentar um Schwannoma vestibular intracanalicular.

\section{Grupo CT: Indivíduos controle (Tabela 1)}

Vinte orelhas de 10 pessoas, quatro mulheres e seis homens, com idades entre 23 e 28 anos. Os critérios de inclusão observados e sugeridos por American ECG Society, $1994^{10}$, foram: ausência de sintomas otoneurológicos ou neurológicos; abstenção de drogas, álcool e fumo nos três dias antecedentes ao teste; otoscopia dentro dos padrões da normalidade; limiares à audiometria tonal inferiores a 15 dB nível de audição nas freqüências de 250, 500, 1.000, 2.000, 3.000, 4.000, 6.000 e 8.000 Hz, bilateralmente; índice percentual de reconhecimento de fala igual ou superior a 96\%, bilateralmente; curvas timpanométricas tipo A, bilateralmente; presença dos reflexos do estapédio ipsi e contralaterais nas freqüências de 500, 1.000, 2.000 e 4.000 $\mathrm{Hz}$, bilateralmente; assinar o termo de consentimento pósinformação.

\section{Grupo CH: Indivíduos com diagnóstico clínico definido de hydrops endolinfático (Tabela 2)}

Vinte orelhas de 17 pacientes, 13 mulheres e 4 homens. Os critérios de inclusão, segundo sugestões do Committee on Hearing and Equilibrium, 1995², quanto ao diagnóstico definido de doença de Menière, foram: possuir história de dois ou mais episódios espontâneos de vertigem rotatória e episódica, com duração de 20 minutos ou mais; referir zumbido ou sensação de ouvido cheio no lado afetado 
Tabela 1. Indivíduos do grupo controle (CT), quanto ao sexo, idade em anos, amplitude em microvolts entre a linha de base e o primeiro pico, e entre a linha de base e o segundo pico, do segundo componente do potencial de ação (I/BSL e II/BSL) e relação entre as amplitudes do potencial de somação e ação (SP/AP).

\begin{tabular}{|c|c|c|c|c|c|}
\hline $\mathrm{N}$ & Sexo & Idade (anos) & I/BSL $(\mu \mathrm{V})$ & II/BSL $(\mu \mathrm{V})$ & SP/AP \\
\hline 1 & $M$ & 27 & $-0,16$ & 0,24 & 0,28 \\
\hline 3 & $\mathrm{~F}$ & 28 & 0,30 & 1,37 & 0,21 \\
\hline 5 & M & 28 & $-0,33$ & 0,67 & 0,25 \\
\hline 6 & - & - & $-0,12$ & 0,41 & 0,23 \\
\hline 7 & $\mathrm{~F}$ & 24 & $-0,67$ & $-0,64$ & 0,34 \\
\hline 8 & - & - & 0,43 & 0,31 & 0,24 \\
\hline 9 & M & 27 & 0,61 & 1,42 & 0,29 \\
\hline 10 & - & - & 4,68 & 1,64 & 0,18 \\
\hline 11 & $\mathrm{~F}$ & 26 & $-2,68$ & $-0,07$ & 0,17 \\
\hline 12 & - & - & $-12,07$ & $-3,76$ & 0,10 \\
\hline 13 & $\mathrm{~F}$ & 28 & $-0,40$ & 0,01 & 0,27 \\
\hline 14 & - & - & $-0,33$ & 0,45 & 0,20 \\
\hline 15 & M & 26 & $-0,17$ & 2,11 & 0,11 \\
\hline 16 & - & - & $-0,54$ & 1,48 & 0,17 \\
\hline 17 & M & 23 & $-0,03$ & 2,78 & 0,12 \\
\hline 18 & - & - & 4,28 & 6,81 & 0,20 \\
\hline 19 & M & 28 & $-0,06$ & 0,71 & 0,23 \\
\hline 20 & - & - & $-0,50$ & 0,56 & 0,27 \\
\hline
\end{tabular}

Legenda: N - número de ouvidos examinados; M - masculino; F- feminino; I/BSL - amplitude entre a linha de base e o primeiro pico do segundo componente do potencial de ação; II/BSL - amplitude entre a linha de base e o segundo pico do segundo componente do potencial de ação; SP/AP - relação entre as amplitudes do potencial de somação e ação; $\mu \mathrm{V}$ - microvolts.

Observação: Valores negativos indicam que o primeiro ou segundo pico situou-se abaixo da linha de base. Células preenchidas pelo hífen(-) significam que foram avaliados os dois ouvidos de um mesmo indivíduo.

Tabela 2. Grupo de portadores de labirintopatias sem evidência clínica de hydrops endolinfático (SH), quanto ao sexo, idade em anos, amplitude em microvolts entre a linha de base e o primeiro pico, e entre a linha de base e o segundo pico, do segundo componente do potencial de ação (I/BSL, II/BSL) e relação entre as amplitudes do potencial de somação e ação (SP/AP).

\begin{tabular}{|c|c|c|c|c|c|}
\hline $\mathrm{N}$ & Sexo & Idade (anos) & $\mathrm{I} / \mathrm{BSL}(\mu \mathrm{V})$ & II/BSL $(\mu \mathrm{V})$ & $S P / A P$ \\
\hline 1 & $\bar{M}$ & 80 & $-0,88$ & $-0,02$ & 0,48 \\
\hline 2 & $\mathrm{~F}$ & 30 & 0,13 & 0,08 & 0,25 \\
\hline 3 & $\mathrm{~F}$ & 60 & $-0,27$ & 0,21 & 0,54 \\
\hline 4 & - & - & $-0,31$ & 0,75 & 0,44 \\
\hline 5 & $\mathrm{~F}$ & 81 & 0,33 & 0,96 & 0,47 \\
\hline 6 & - & - & $-0,06$ & 0,73 & 0,36 \\
\hline 7 & $\mathrm{~F}$ & 61 & 0,58 & 2,25 & 0,15 \\
\hline 8 & - & - & 0,19 & $-0,04$ & 0,49 \\
\hline 9 & $\mathrm{~F}$ & 36 & 3,66 & 2,75 & 0,14 \\
\hline 10 & $\mathrm{~F}$ & 40 & $-1,35$ & $-1,31$ & 0,62 \\
\hline 11 & - & - & 0,98 & 1,71 & 0,43 \\
\hline 12 & $\mathrm{~F}$ & 64 & 0,34 & 0,25 & 0,2 \\
\hline 13 & M & 62 & 0,4 & 0,92 & 0,38 \\
\hline 14 & $M$ & 15 & 0,08 & 1,46 & 0,36 \\
\hline 15 & - & - & $-0,5$ & $-0,66$ & 0,56 \\
\hline 16 & $\mathrm{~F}$ & 48 & 0,2 & 0,84 & 0,34 \\
\hline 17 & $\mathrm{~F}$ & 52 & 1,29 & 2,5 & 0,3 \\
\hline 18 & $M$ & 64 & $-1,54$ & $-0,34$ & 0,54 \\
\hline 19 & $\mathrm{~F}$ & 61 & $-0,21$ & 0,32 & 0,46 \\
\hline 20 & $\mathrm{~F}$ & 30 & 2,5 & 4,67 & 0,2 \\
\hline
\end{tabular}

Legenda: $\mathrm{N}$ - número de ouvidos examinados; M - masculino; F- feminino; I/BSL - amplitude entre a linha de base e o primeiro pico do segundo componente do potencial de ação; II/BSL - amplitude entre a linha de base e o segundo pico do segundo componente do potencial de ação; SP/AP - relação entre as amplitudes do potencial de somação e ação; $\mu \mathrm{V}$ - microvolts. Observação: Valores negativos indicam que o primeiro ou segundo pico situou-se abaixo da linha de base. Células preenchidas pelo hífen(-) significam que foram avaliados os dois ouvidos de um mesmo indivíduo. 
Tabela 3. Grupo de pacientes com diagnóstico clínico definido de hydrops endolinfático ( $\mathrm{CH}$ ), quanto ao sexo, idade em anos, amplitude em microvolts entre a linha de base e o primeiro pico, e entre a linha de base e o segundo pico, do segundo componente do potencial de ação (I/BSL, II/BSL) e relação entre as amplitudes do potencial de somação e ação (SP/AP).

\begin{tabular}{|c|c|c|c|c|c|}
\hline $\mathrm{N}$ & Sexo & Idade (anos) & $\mathrm{I} / \mathrm{BSL}(\mu \mathrm{V})$ & II/BSL $(\mu \mathrm{V})$ & SP/AP \\
\hline 1 & $M$ & 56 & -6 & 0,5 & 0,38 \\
\hline 3 & - & - & $-0,05$ & 0,08 & 0,49 \\
\hline 5 & $\mathrm{~F}$ & 45 & 1,71 & 1,62 & 0,46 \\
\hline 6 & $M$ & 32 & $-0,52$ & 0,21 & 0,47 \\
\hline 7 & $M$ & 36 & $-0,06$ & 0,27 & 0,71 \\
\hline 10 & - & - & $-0,4$ & 2,06 & 0,52 \\
\hline 11 & $\mathrm{~F}$ & 31 & 1 & 1 & 0,55 \\
\hline 12 & $\mathrm{~F}$ & 47 & 0,67 & 2,33 & 0,46 \\
\hline 13 & $\mathrm{~F}$ & 42 & $-0,58$ & 0,88 & 0,42 \\
\hline 14 & $M$ & 62 & $-0,46$ & 0,46 & 0,73 \\
\hline 15 & - & - & 0,64 & 1,52 & 0,37 \\
\hline
\end{tabular}

Legenda: $\mathrm{N}$ - número de ouvidos examinados; $\mathrm{M}$ - masculino; F- feminino; l/BSL - amplitude entre a linha de base e o primeiro pico do segundo componente do potencial de ação; II/BSL - amplitude entre a linha de base e o segundo pico do segundo componente do potencial de ação; SP/AP - relação entre as amplitudes do potencial de somação e ação; $\mu \mathrm{V}$ - microvolts. Observação: Valores negativos indicam que o primeiro ou segundo pico situou-se abaixo da linha de base. Células preenchidas pelo hífen(-) significam que foram avaliados os dois ouvidos de um mesmo indivíduo.

e ter perda auditiva documentada. Foram também critérios de inclusão: otoscopia dentro dos padrões da normalidade; limiares audiométricos iguais ou maiores que $50 \mathrm{~dB}$ nível de audição, calculados pela média das freqüências 500, 1.000, $2.000,3.000$ e $4000 \mathrm{~Hz}$; curvas timpanométricas tipo A, bilateralmente; ausência de doenças neurológicas e/ou otoneurológicas concomitantes; assinar o termo de consentimento pós-informação.

\section{Grupo SH: Pacientes com labirintopatias sem evidência clínica de hydrops endolinfático (Tabela 3)}

Vinte orelhas de 15 pacientes, 11 mulheres e 4 homens. Os critérios para inclusão neste grupo foram: história de crises de tonturas sem as características típicas da vertigem episódica da doença de Menière; não relatar sensação de plenitude aural, pressão na cabeça e zumbido flutuante; otoscopia dentro dos padrões da normalidade; ter perda auditiva documentada e limiares audiométricos iguais ou maiores que $50 \mathrm{~dB}$ nível de audição, calculados pela média das freqüências 500, 1.000, 2.000, 3.000 e 4000 Hz; curvas timpanométricas tipo A, bilateralmente; ausência de doenças neurológicas e/ou otoneurológicas centrais concomitantes; assinar o termo de consentimento pós-informação.

Todos os exames foram realizados em equipamento Navigator $\mathrm{SE}^{\circledR}$ da Bio-logical Systems Corp, seguindo o mesmo protocolo.
O examinador demarcou os pontos BSL, SP, AP, I e II e o equipamento foi programado para fornecer as amplitudes em $\mu V$ entre: SP e a BSL; AP e a BSL; I e a BSL e II e a BSL (Figura 1) e calcular a relação SP/AP.

$\mathrm{Na}$ análise estatística foi calculada a média das idades, em anos, nos três grupos e a distribuição percentual dos grupos quanto ao sexo dos pacientes.

Os parâmetros SP/AP, I/BSL, II/BSL foram examinados estatisticamente e a análise de variância ANOVA foi empregada para averiguar diferenças de comportamento entre os grupos. O nível de significância estabelecido foi $5,0 \%$ e os valores acima deste limite foram identificados por um asterisco (Tabelas 4, 5 e 6).

As diferenças significantes foram avaliadas por meio do teste de comparações múltiplas de Bonferroni com o intuito de identificar os grupos de comportamentos desiguais ${ }^{11}$. Foram dispostos os correspondentes gráficos dos intervalos de confiança (Gráficos 1, 2 e 3). Os intervalos de confiança apresentados foram calculados pela fórmula: intervalo de confiança $=$ média $\pm 1,96 *$ desvio-padrão $/ \sqrt{ }$ (n-1).

Com a finalidade de medir a capacidade de decisão dos parâmetros, calcularam-se os índices de sensibilidade e especificidade entre os grupos Com hydropse Sem bydrops, para os dados que foram considerados estatisticamente significantes. 


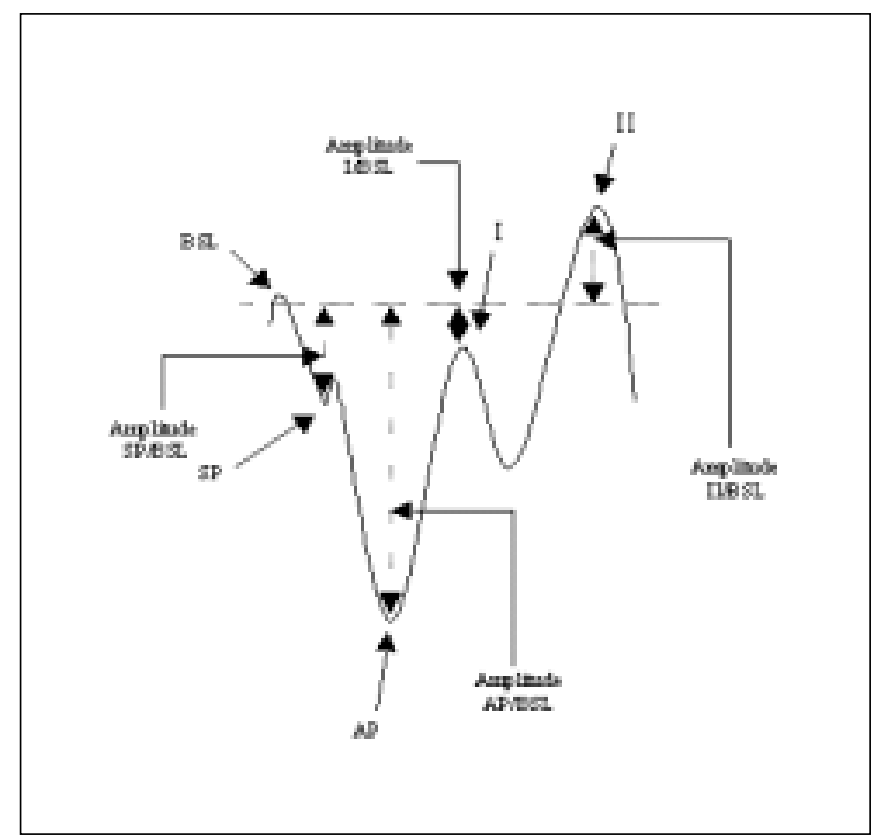

Figura 1. Registro da EcochG TT. O esquema mostra a demarcação da linha de base (BSL), potencial de somação (SP), potencial de ação (AP), picos I e II, realizadas pelo examinador e as amplitudes SP/BSL, AP/BSL, I/BSL e II/BSL fornecidas pelo software.

A sensibilidade foi definida como a capacidade da medida em identificar os pacientes do grupo com diagnóstico clínico definido de hydrops e a especificidade como a capacidade da medida em identificar os indivíduos do grupo sem evidência clínica de bydrops.

A eficiência dos parâmetros foi calculada seguindo as instruções preconizadas por Silva ${ }^{12}$. A eficiência teve como objetivo a possibilidade da sensibilidade (S) e especificidade (E) possuírem valores similares e próximos de um (100,0\%). $O$ valor de maior equilíbrio entre a sensibilidade e a especificidade para identificar o grupo com hydrops foi destacado em negrito (Tabela 7).

\section{RESULTADOS}

A média de idade do grupo controle foi inferior a dos outros grupos ( $\mathrm{CT}=26,50$ anos; $\mathrm{SH}=52,27$ anos e $\mathrm{CH}=48$ anos). Houve prevalência do sexo masculino no grupo controle (12:8); nos outros dois grupos $75 \%$ dos pacientes eram do sexo feminino.

A análise de variância ANOVA e o teste de comparações múltiplas de Bonferroni demonstraram que os três grupos apresentaram comportamentos estatísticos diferentes quando se analisou a relação SP/AP, mas não houve diferenças estatísticas significativas entre os três grupos quando os parâmetros avaliados foram a I/BSL e II/BSL (Tabelas 4, 5 e 6 e Gráficos 1, 2 e 3).
Tabela 4. Média e desvio-padrão, em microvolts, da amplitude entre a linha de base e o primeiro pico que delimita o segundo componente do potencial de ação (I/BSL), nos grupos CT (controle), $\mathrm{CH}$ (diagnóstico clínico definido de hydrops endolinfático) e SH (ouvidos sem evidência clínica de hydrops endolinfático).

\begin{tabular}{lclc}
\hline Estatística & $\mathrm{CT}$ & $\mathrm{SH}$ & $\mathrm{CH}$ \\
\hline Média & $-0,26$ & 0,28 & $-0,33$ \\
Desvio-padrão & 3,22 & 1,19 & 1,59 \\
\hline
\end{tabular}

Legenda: CT-grupo controle; SH-grupo de indivíduos portadores de labirintopatias sem evidência clínica de hydrops endolinfático; $\mathrm{CH}$ grupo dos indivíduos com diagnóstico clínico definido de hydrops endolinfático.

\begin{tabular}{lcccc} 
ANOVA & & & \\
\hline $\begin{array}{l}\text { Fonte de } \\
\text { Variação }\end{array}$ & $\begin{array}{c}\text { Graus de } \\
\text { Liberdade }\end{array}$ & $\begin{array}{c}\text { Quadrado } \\
\text { Médio }\end{array}$ & F & $\begin{array}{c}\text { Nível de } \\
\text { significância }\end{array}$ \\
\hline $\begin{array}{l}\text { Efeito } \\
\text { Erro }\end{array}$ & 2 & 2,221 & 0,466 & 0,630 \\
\hline GRUPO COM HYDROPS $(\mathrm{CH})=$ GRUPO & SEM HYDROPS $(\mathrm{SH})=$ \\
GRUPO CONTROLE $(\mathrm{CT})$.
\end{tabular}

Tabela 5. Média e desvio-padrão, em microvolts, da amplitude entre a linha de base e o primeiro pico que delimita o segundo componente do potencial de ação (II/BSL), nos grupos CT (controle), $\mathrm{CH}$ (diagnóstico clínico definido de hydrops endolinfático) e SH (ouvidos sem evidência clínica de hydrops endolinfático).

\begin{tabular}{lccc}
\hline Estatística & $\mathrm{CT}$ & $\mathrm{SH}$ & $\mathrm{CH}$ \\
\hline Média & 1,03 & 0,90 & 1,03 \\
Desvio-padrão & 1,94 & 1,19 & 0,98 \\
\hline
\end{tabular}

Legenda: CT-grupo controle; SH-grupo de indivíduos portadores de labirintopatias sem evidência clínica de hydrops endolinfático; $\mathrm{CH}$ grupo dos indivíduos com diagnóstico clínico definido de hydrops endolinfático.

\begin{tabular}{lcccc} 
ANOVA & & & \\
\hline $\begin{array}{l}\text { Fonte de } \\
\text { Variação }\end{array}$ & $\begin{array}{c}\text { Graus de } \\
\text { Liberdade }\end{array}$ & $\begin{array}{c}\text { Quadrado } \\
\text { Médio }\end{array}$ & $\mathrm{F}$ & $\begin{array}{c}\text { Nível de } \\
\text { significância }\end{array}$ \\
\hline Efeito & 2 & 0,107 & 0,049 & 0,952 \\
Erro & 57 & 2,195 & & \\
\hline GRUPO COM HYDROPS $(\mathrm{CH})=$ GRUPO & SEM HYDROPS $(\mathrm{SH})=$ \\
GRUPO CONTROLE (CT) & & &
\end{tabular}

Quando se analisou os valores da SP/AP entre os grupos com diagnóstico clínico definido de bydrops $(\mathrm{CH}) \mathrm{e}$ sem evidências clínicas de hydrops (SH) para calcular os índices de sensibilidade e especificidade o valor 0,46 foi o que demonstrou maior equilíbrio entre a sensibilidade e a especificidade (Tabela 7).

A avaliação conjunta das 3 informações não foi possível, pois as informações I/BSL e II/BSL não apresentaram comportamento estatístico diferente nas comparações entre os grupos. 
Tabela 6. Média e desvio-padrão, da relação percentual entre as amplitudes de somação e de ação (SP/AP), nos grupos CT (controle), $\mathrm{CH}$ (diagnóstico clínico definido de hydrops endolinfático) e SH(ouvidos sem evidência clínica de hydrops endolinfático).

\begin{tabular}{lccc}
\hline Estatística & $\mathrm{CT}$ & $\mathrm{SH}$ & $\mathrm{CH}$ \\
\hline Média & 0,22 & 0,39 & 0,52 \\
Desvio-padrão & 0,06 & 0,14 & 0,11 \\
\hline
\end{tabular}

Legenda: CT-grupo controle; SH-grupo de indivíduos portadores de labirintopatias sem evidência clínica de hydrops endolinfático; $\mathrm{CH}$ grupo dos indivíduos com diagnóstico clínica definido de hydrops endolinfático.

ANOVA

\begin{tabular}{lcccc}
\hline $\begin{array}{l}\text { Fonte de } \\
\text { Variação }\end{array}$ & $\begin{array}{c}\text { Graus de } \\
\text { Liberdade }\end{array}$ & $\begin{array}{c}\text { Quadrado } \\
\text { Médio }\end{array}$ & $\mathrm{F}$ & $\begin{array}{c}\text { Nível de } \\
\text { significância }\end{array}$ \\
\hline Efeito & 2 & 0,455 & 36,572 & $<0,001^{*}$ \\
Erro & 57 & 0,012 & & \\
\hline
\end{tabular}

GRUPO COM HYDROPS $(\mathrm{CH})>$ GRUPO SEM HYDROPS $(\mathrm{SH})>$ GRUPO CONTROLE (CT)

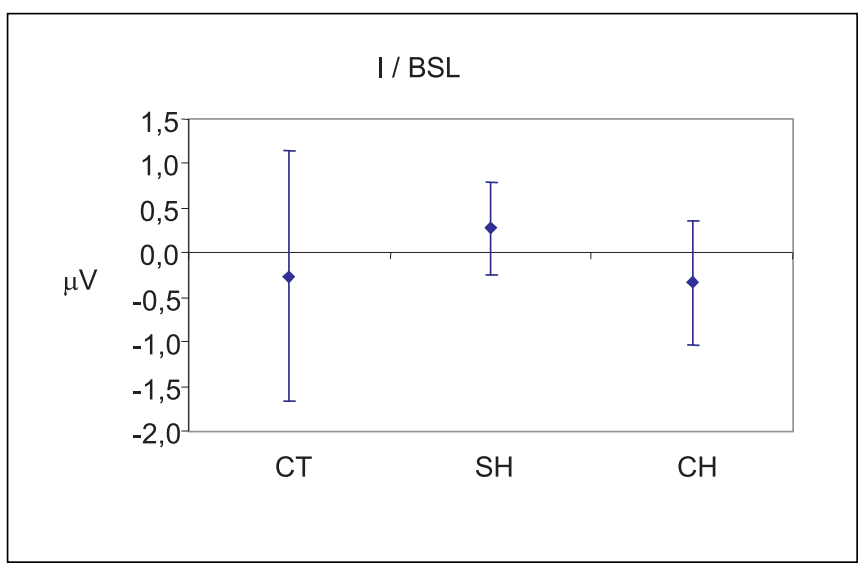

Gráfico 1. Gráfico demonstrativo do intervalo de confiança, contendo a média expressa em microvolts, da amplitude entre a linha de base e o primeiro pico que delimita o segundo componente do potencial de ação (I/BSL).

Legenda: CT-grupo controle; SH-grupo de indivíduos portadores de labirintopatias sem evidência clínica de hydrops endolinfático; $\mathrm{CH}$ grupo dos indivíduos com diagnóstico clínico definido de hydrops endolinfático.

\section{DISCUSSÃO}

Portmann relatou o grande desafio que é o estudo da doença de Menière e de tudo o que a ela está relacionado quando a definiu como doença de grande variabilidade de apresentação clínica, de diagnóstico impreciso e de tratamento ineficaz. ${ }^{13}$ Todas estas características ainda aliadas
II / BSL

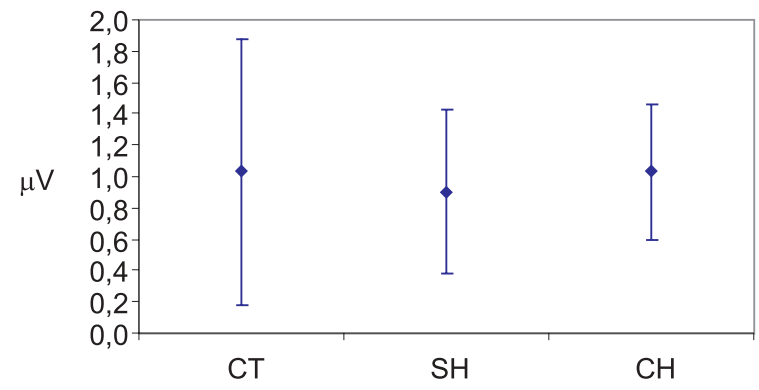

Gráfico 2. Gráfico demonstrativo do intervalo de confiança, contendo a média expressa em microvolts, da amplitude entre a linha de base e o segundo pico que delimita o segundo componente do potencial de ação (II/BSL).

Legenda: CT-grupo controle; SH-grupo de indivíduos portadores de labirintopatias sem evidência clínica de hydrops endolinfático; $\mathrm{CH}$ grupo dos indivíduos com diagnóstico clínico definido de hydrops endolinfático.

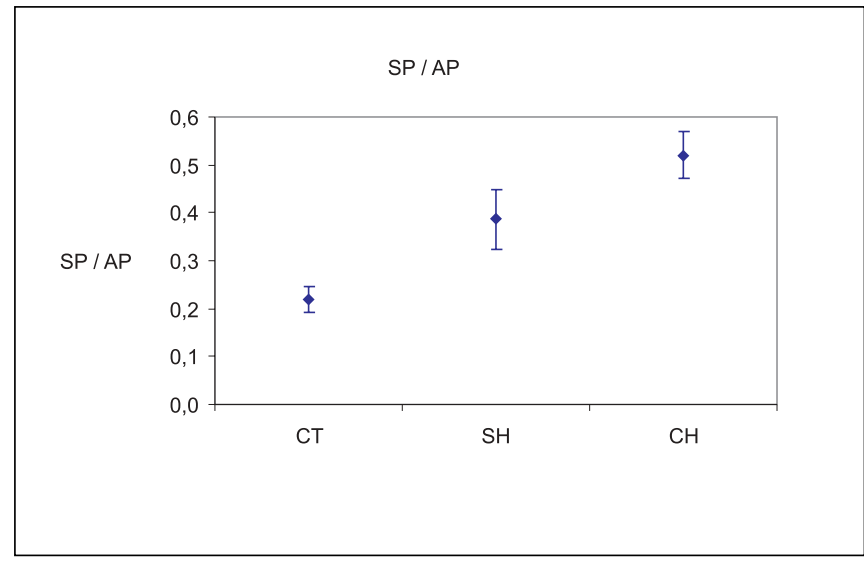

Gráfico 3. Gráfico demonstrativo do intervalo de confiança, contendo a média expressa em microvolts, da relação SP/AP.

Legenda: CT-grupo controle; SH-grupo de indivíduos portadores de labirintopatias sem evidência clínica de hydrops endolinfático; $\mathrm{CH}$ grupo dos indivíduos com diagnóstico clínico definido de hydrops endolinfático.

a uma alta prevalência ${ }^{14}$ tornam as pesquisas sobre o assunto sempre justificáveis.

As dificuldades no diagnóstico de certeza, que só pode ser feito por meio de estudo histológico após a morte, têm levado ao desenvolvimento de vários critérios clínicos para o estabelecimento do diagnóstico da doença. Todos estes critérios enfatizam a associação de sinais e sintomas clínicos 
com achados audiométricos. ${ }^{2}$

A eletrococleografia é o exame mais empregado para o diagnóstico do bydrops endolinfático e vários trabalhos comprovam sua eficácia na detecção das alterações da eletrofisiologia da orelha interna ${ }^{15,16}$. Porém são muito diferentes os relatos encontrados na literatura sobre os parâmetros ideais, sobre os valores destes parâmetros para o diagnóstico do bydrops endolinfático e sobre a especificidade e sensibilidade do método no diagnóstico da doença de Menière. ${ }^{17,18}$

$\mathrm{O}$ estudo em pacientes com limiares auditivos maiores ou iguais a $50 \mathrm{~dB}$ se justifica pela alta prevalência deste grau de severidade da perda auditiva nos pacientes com bydrops, principalmente nas fases mais avançadas da doença.

Tabela 7. Índices de sensibilidade e especificidade dos valores da relação SP/AP estabelecidos entre o grupo $\mathrm{SH}$ (ouvidos sem evidência clínica de hydrops endolinfático) e $\mathrm{CH}$ (diagnóstico clínico definido de hydrops endolinfático).

\begin{tabular}{ccc}
\hline SP/AP & Sensibilidade & Especificidade \\
\hline 0,14 & 1,000 & 0,000 \\
0,15 & 1,000 & 0,050 \\
0,20 & 1,000 & 0,100 \\
0,25 & 1,000 & 0,200 \\
0,30 & 1,000 & 0,250 \\
0,34 & 1,000 & 0,300 \\
0,36 & 1,000 & 0,350 \\
0,37 & 1,000 & 0,450 \\
0,38 & 0,900 & 0,450 \\
0,40 & 0,850 & 0,500 \\
0,42 & 0,800 & 0,500 \\
0,43 & 0,750 & 0,500 \\
0,44 & 0,750 & 0,550 \\
0,46 & 0,750 & 0,600 \\
0,47 & 0,600 & 0,650 \\
0,48 & 0,550 & 0,700 \\
0,49 & 0,550 & 0,750 \\
0,50 & 0,500 & 0,800 \\
0,52 & 0,450 & 0,800 \\
0,53 & 0,400 & 0,800 \\
0,54 & 0,300 & 0,800 \\
0,55 & 0,300 & 0,900 \\
0,56 & 0,250 & 0,900 \\
0,62 & 0,250 & 0,950 \\
0,64 & 0,250 & 1,000 \\
0,67 & 0,200 & 1,000 \\
0,68 & 0,150 & 1,000 \\
0,71 & 0,100 & 1,000 \\
0,73 & 0,050 & 1,000 \\
\hline
\end{tabular}

Legenda: SP/AP - relação percentual entre a amplitude do potencial de somação e do potencial de ação. Em negrito o valor de maior equilíbrio entre a sensibilidade e a especificidade $(S=E=1)$.
O nível de audição igual ou superior a $50 \mathrm{~dB}$ foi escolhido por estudar a influência do AP na relação SP/ AP. O AP seria um potencial influenciável pela destruição de células ciliadas, encontrada em perdas auditivas superiores a 50 decibéis e pela adaptação, presente em doenças que envolvam o nervo auditivo e/ou as vias auditivas do tronco encefálico. ${ }^{5,19}$

A destruição de células ciliadas e a adaptação alterariam a amplitude do AP determinando alteração da relação SP/AP, não por aumento do SP, mas por diminuição do AP. Esta redução se justifica pela falta de sincronia nos disparos dos neurônios formadores do $\mathrm{AP}^{7}$ e foi observada por outros autores. ${ }^{8}$

A eletrococleografia transtimpânica foi o método de exame utilizado nesta pesquisa, por ser consagrada pela literatura como a que permite a obtenção das melhores respostas, com pouca variabilidade intrateste e interteste. ${ }^{20,21}$

A escolha da relação SP/AP como principal parâmetro na avaliação dos resultados foi fundamentada na afirmação de Coats que propôs a avaliação desta relação para diminuir a grande variabilidade interindividual das amplitudes de SP e $\mathrm{AP}^{22}$.

A avaliação das amplitudes dos dois picos que delimitam o $\mathrm{N}_{2}$ (I/BSL e II/BSL) se baseou na hipótese de que a associação destes parâmetros com a relação SP/AP poderia ajudar na diferenciação entre os grupos, seguindo o conceito de associação de parâmetros bastante utilizado no estabelecimento do diagnóstico clínico da doença ${ }^{23}$.

A inclusão do grupo com labirintopatia sem evidência clínica de bydrops surgiu da necessidade de comparação de grupos com limiares auditivos semelhantes, pois a hipótese testada seria a alteração da relação SP/AP pela diminuição do AP, em pacientes com destruição de células cililadas e, para que isto fosse possível, tomou-se enorme cuidado na exclusão de pacientes com doenças neurológicas e otoneurológicas centrais.

A média da idade do grupo com bydrops foi de 48 anos. Esta idade se situa dentro da faixa etária de maior prevalência da doença relatada pela literatura. ${ }^{24}$

A prevalência do sexo feminino no grupo sem bydrops e com bydrops era esperada por serem as labirintopatias mais freqüentes nas mulheres. ${ }^{14,24}$

Não houve diferenças significativas entre os grupos quanto a I/BSL e II/BSL que delimitam $\mathrm{O} \mathrm{N}_{2}$ do AP, contrariando os achados Munhoz $^{23}$ que estudou esta associação, porém, em pacientes com limiares audiométricos inferiores a 50 decibéis, obtendo incremento da sensibilidade e especificidade do método com a análise combinada entre os valores da SP/AP e os da I/BSL. A amplitude de $\mathrm{N}_{2}$ já havia sido estudada por outros autores, sem a obtenção de significância para o diagnóstico 
diferencial das labirintopatias. ${ }^{7}$

Os três grupos apresentaram comportamentos diferentes quando se avaliou a relação SP/AP. O grupo controle apresentou comportamento muito diferente dos outros dois grupos e não foi utilizado na continuação da análise estatística por ser apenas um grupo controle. O maior interesse era conseguir valores capazes de diferenciar laboratorialmente os pacientes do grupo com diagnóstico definido de bydrops dos pacientes do grupo sem evidências clínicas de hydrops, pela relevância clínica destes dois grupos.

Analisando os grupos com diagnóstico definido de hydrops e sem evidências clínicas de hydrops, o valor da relação SP/AP 0,46 foi o que mostrou maior equilíbrio entre a sensibilidade e a especificidade (sensibilidade de $75 \%$ e especificidade de 60\%). Não encontramos na literatura estudos específicos sobre pacientes com limiares iguais ou maiores que 50 decibéis para que pudéssemos comparar os resultados, porém vários estudos já haviam registrado a influência da perda auditiva nos achados eletrococleográficos. ${ }^{5,25}$ Alguns autores não relataram dificuldades para o estabelecimento do diagnóstico diferencial entre grupos semelhantes aos deste estudo, quando analisaram a relação $\mathrm{SP} / \mathrm{AP}^{26}$ porém, Coats $^{22}$ também não conseguiu diferenciação entre os grupos quando analisou somente a relação SP/AP.

Munhoz $^{23}$ registrou índices de sensibilidade e especificidade de $100 \%$ na determinação da presença de hydrops endolinfático quando utilizou o valor 0,35 para a relação SP/AP usando a mesma técnica proposta nesta pesquisa, porém ele estudou pacientes com limiares até 50 decibéis. Nosso estudo demonstrou a extrema importância da diferenciação entre pacientes com limiares abaixo e acima de 50 decibéis, pois o exame perde muito em sensibilidade e especificidade no grupo de pacientes com limiares acima de 50 decibéis e serviu ainda para justificar a grande variabilidade dos índices gerais de sensibilidade e especificidade da relação SP/AP para o diagnóstico do hydrops endolinfático encontrados na literatura, visto que estes dois grupos nunca foram estudados separadamente.

A questão do diagnóstico eletrococleográfico nos pacientes com limiares auditivos maiores ou iguais a 50 dB não foi resolvida pela associação de parâmetros proposta neste estudo (SP/AP, I/BSL e II/ BSL), mas a solução pode estar no uso de técnicas diferentes, com estímulos que permitam maior seletividade de freqüência $^{18,27,28}$ ou até mesmo na mudança da maneira de avaliação dos parâmetros obtidos no exame. ${ }^{22}$ Sass ressaltou a eficiência diagnóstica da eletrococleografia com tone burst de $1 \mathrm{KHz}$. A associação desta técnica à avaliação da SP/ AP evocada por click elevou a sensibilidade no diagnóstico do bydrops endolinfático de $62,0 \%$ para $82,0 \%$ sem alteração da especificidade ${ }^{18}$.

\section{CONCLUSÕES}

1. Os três grupos apresentaram comportamentos estatísticos diferentes entre si.

2. A relação SP/AP foi o parâmetro mais sensível e específico para identificação dos pacientes com diagnóstico clínico definido de bydrops endolinfático.

3. A associação dos parâmetros não foi possível e portanto não foi útil para a caracterização laboratorial do grupo com diagnóstico clínico definido de bydrops endolinfático.

\section{REFERÊNCIAS BIBLIOGRÁFICAS}

1. Alford BR. Report of Subcommitte on Equilibrium and its Measurements. Menière's disease: criteria for diagnosis and evaluation of therapy for reporting results. Trans Am Acad Ophthalmol Otolaryngol 1972;76:1462-4.

2. Committee on Hearing and Equilibrium. Committee on Hearing and Equilibrium guidelines for the diagnosis and evaluation of therapy in Meniere's disease. Otolaryngol Head Neck Surg 1995;113:181-85.

3. Munhoz MSL, Silva MLG, Ganança MM, Caovilla HH, Frazza MM. Eletrococleografia. In: Munhoz MSL, Caovilla HH, Silva MLG, Ganança MM (editores). Audiologia clínica. São Paulo: Atheneu; 2000b. p. 173-190.

4. Eggermont JJ. Basic principles for Electrocochleography Acta Otolaryngol Suppl 1974;316:7-16.

5. Eggermont JJ. Summating potentials in Meniere's disease. Arch Otorhinolaryngol 1979;222:63-75.

6. Staller SJ, Goin DW, Asher DL, Mischke RE. Summating potential in Meniere's disease. Laryngoscope 1982 Dec;92(12):1383-9.

7. Marangos N, Laszig R. Differential diagnosis of enhanced summating to compound action potential ratio. In: Arenberg IK (editor). Surgery of the Inner Ear. Amsterdam: Kugler; 1991. p. 263-7.

8. Levine SC, Margolis RH, Fournier EM, Winzenburg SM. Tympanic electrocochleography for evaluation of endolymphatic bydrops. Laryngoscope 1992 Jun;102:614-22.

9. Arenberg IK, Gibson WPR, Höhmann D, Mihalco LL. International standards for transtympanic electrocochleography. In: Höhmann (editor). ECoG, OAE and intraoperative monitoring. Amsterdam: Klugler; 1993. p. 1158 .

10. American ECG Society. Clinical evoked potentials guide lines. Recommended standards for normative studies of evoked potentials, statistical analysis of results and criteria for clinically significant abnormality. J Clin Neurophysiol 1994;11:47-7.

11. Downing D, Clark J. Estatística aplicada. São Paulo: Saraiva; 1992.

12. Silva MCM. Testes de diagnóstico ou screening tests. In: Silva MCM (autora). Estatística aplicada à psicologia e ciências sociais. Lisboa: McGraw-Hill; 1994. p. 161-180.

13. Potmann G. The old and new in Menière's disease - over 60 years in retrospect and a look to the future. Otolaryngologic Clinics of North America 1980;13(4):567-75.

14. Anadão CA. Doença de Menière: revisão. Acta Awho 1993;12(2):4450 .

15. Gatland DJ, Billings RJ, Youngs RP, Johnson NP. Investigation of the physiological basis of summating potential changes in endolymphatic hydrops. Acta Otolaryngol 1988 Mar-Apr;105 (34):218-22.

16. Orchik DJ, Shea JJ, Ge NN. Summating potential and action potential ratio in Meniere's disease before and after treatment. Am J Otol 1998 Jul;19(4):478-82. 
17. Dornhoffer JL. Diagnosis of cochlear Menière's disease with electrocochleography. ORL 1998;60:301-05.

18. Sass K. Sensitivity and specificity of transtympanic electrocochleography in Meniere's disease. Acta Otolaryngol 1998;118:1506.

19. Ozdamar O, Dallos P. Synchronous responses of the primary auditory fibers to the onset of tone burst and their relation to compound action potentials. Brain Res 1978 Oct 20;155(1):16975.

20. Densert B, Arlinger S, Sass K, Hergils L. Reproducibility of the electric response components in clinical electrocochleography. Audiology 1994;33:254-63.

21. Roland PS, Rosenbbloom J, Yellin W, Meyerhoff W. Intrasubject tese-retest variability in clinical eletrococheography. Laryngoscope 1993;103:963-66.

22. Coats AC. The summating potential and Meniere's disease. I. summating potential amplitude in Meniere and non-Meniere ears. Arch Otolaryngol 1981 Apr;107(4):199-208.
23. Munhoz MSL. Da sensibilidade e especificidade da eletrococleografia transtimpânica em pacientes com e sem hydrops endolinfático (tese). São Paulo: Universidade Federal de São Paulo - Escola Paulista de Medicina; 2001.

24. Campos CAH. Principais quadros clínicos no adulto e no idoso. In Ganança MM (editor). Vertigem tem cura? São Paulo: Lemos editorial 1998. p.49-57.

25. Ferraro J, Lovar GB, Arenberg IK. The use of eletrocochleography in the diagnosis, assessment and monitoring of endolymphatic hydrops. Otolaryngol Clin N Am 1983;16(1):69-82.

26. Filipo R, Barbara M. Natural history of Menière's disease: staging the patients or their symptoms? Acta Otolaryngol (Stockh) 1997;526 Suppl:10-3.

27. Conlon BJ, Gibson WPR. Electrocochleography in the diagnosis of Meniere's disease. Acta Otolaryngol 2000 Jun;120(4):480-3.

28. Sass K, Densert B, Magnusson M, Whitaker S. Electrocochleographic signal analysis: condensation and rarefaction click stimulation contributes to diagnosis in Menière's disorder. Audiology 1998;37:198-206. 